\title{
Reflexiones en torno al diseño de una propuesta formativa sobre variación lineal orientada a futuros profesores de secundaria
}

\author{
María Teresa Dávila Araiza' ${ }^{1}$ y Karina Jaquelin Herrera Garcia² \\ e-mail: ${ }^{1}$ maria.davila@unison.mx, ${ }^{2}$ jaquelin_herrera@hotmail.es \\ Universidad de Sonora
}

\section{Resumen}

La variación lineal es una noción central en la educación secundaria, que se construye sobre la base de las relaciones de proporcionalidad y abre el camino hacia el estudio de las funciones. Sin embargo, el currículo de secundaria descuida el tratamiento de sus propiedades esenciales, entre ellas las relacionadas con la variación. Este artículo trata sobre una propuesta didáctica realizada en una tesis de maestría en Matemática Educativa para atender la problemática de la enseñanza de la variación lineal en la escuela secundaria, mediante el diseño de actividades didácticas dirigidas a futuros profesores de secundaria para favorecer el desarrollo de conocimientos y competencias didáctico-matemáticos sobre variación lineal. En este escrito, el énfasis se pondrá en describir el proceso de diseño de las actividades didácticas, resaltando el papel que jugó en ello la interacción entre la problemática identificada, las herramientas teóricas del Enfoque Ontosemiótico (EOS) y el diseño de la primera actividad. Aunque el EOS permitió fundamentar el diseño, planificar su implementación y realizar una valoración de su pertinencia a partir de los datos emanados de su puesta en escena, únicamente será discutida la etapa de diseño de las actividades didácticas y su relación con las herramientas teóricas que lo fundamentan.

Palabras clave: Variación lineal, Futuros profesores de Matemáticas, Escuela Secundaria

Recibido 07 de enero de 2021

Aceptado 08 de abril de 2021

\section{Introducción}

En este escrito se describirá el proceso de diseño de la propuesta didáctica del trabajo de tesis de Herrera (2020), presentando algunas reflexiones en torno a la evolución que tuvo el diseño de las actividades, dada la interacción entre la problemática de interés, el referente teórico elegido y la primera actividad didáctica diseñada, hasta llegar al diseño final que se puso en escena con futuros profesores de matemáticas.

El trabajo de Herrera (2020) se orientó hacia la educación secundaria, y la problemática en la cual se enmarca tiene dos ejes principales: la enseñanza de la noción matemática de variación lineal y la formación inicial de profesores de matemáticas de este nivel educativo. En torno a estos dos elementos se diseñaron actividades didácticas cuyo objetivo fue, inicialmente, promover en futuros profesores de matemáticas el desarrollo de conocimientos matemáticos sobre variación lineal; sin embargo, éste se redirigió hacia el desarrollo de Conocimientos y Competencias Didáctico-Matemáticos en torno a esta noción matemática.

Parte de la problemática abordada en la tesis de Herrera tiene una profunda relación con la línea de trabajo de diversos autores, quienes han señalado que el estudio de la variación en los cursos de Cálculo universitarios se desvanece bajo un estudio estático de los números, las variables y las relaciones funcionales, y donde se esconde deliberadamente la relación de estas nociones con los procesos de cambio (Ímaz y Moreno, 2010; Moreno-Armella, 2014; Jiménez, Grijalva, Milner, Dávila-Araiza y Romero, en prensa; Dávila-Araiza y Grijalva, 2020; Jiménez-Rodríguez, 2020). El estudio de la variación lineal en la secundaria 
no escapa de esta perspectiva. Es por ello por lo que el trabajo de Herrera (2020) se enfocó en el desarrollo de un significado variacional de esta noción matemática con futuros profesores de matemáticas de secundaria. Poder clarificar cuál sería dicho significado variacional y cómo articularlo con las directrices curriculares de la escuela secundaria para proponer un diseño didáctico, fueron elementos que motivaron la elección del Enfoque Ontosemiótico (EOS) (Godino, Batanero y Font, 2007) como fundamento teórico para el trabajo.

En un principio, en el trabajo de Herrera (2020) se pretendía diseñar actividades didácticas enfocadas solamente al desarrollo de conocimientos matemáticos; sin embargo, esta idea inicial evolucionó al conjugarse los siguientes elementos: la problemática en torno a la enseñanza de la variación lineal, la inquietud por incidir en el sistema educativo vía la intervención en la formación inicial de los profesores de secundaria, el acceso a herramientas teóricas orientadas al conocimiento y las competencias didácticomatemáticos del profesor y el diseño de la primera actividad didáctica. Para dar cuenta de este proceso de interacción, a continuación, se esbozará la problemática en torno a la enseñanza de la variación lineal y la formación inicial del profesorado mexicano de educación secundaria. Posteriormente, se presentarán los elementos teóricos del EOS empleados para el diseño de la primera de las actividades. Después, se describirá la primera actividad didáctica con base en los elementos teóricos y se explicará cómo se ampliaron los propósitos de las actividades para considerar el desarrollo de Conocimientos y Competencias DidácticoMatemáticos. Finalmente, se describirá la estructura final que se dio al diseño de las actividades y se presentarán algunas reflexiones finales.

\section{La variación lineal y la formación inicial de profesores de secundaria}

La variación lineal es una noción central en la educación secundaria y por demás compleja. En el currículo de la escuela secundaria su estudio inicia sobre la base de las relaciones de proporcionalidad entre dos magnitudes, pero sin restringirse a ellas, y sienta las bases para iniciar el estudio de la noción función y sus diversas representaciones semióticas. Sin embargo, en este proceso, la noción de variación queda desatendida. El currículo y libros de texto favorecen el desarrollo de un significado limitado de la variación lineal, en el sentido de que éste está alejado de su esencia variacional.

Autores como Panorkou y Maloney (2016) afirman que el estudio de la función en la escuela secundaria pone el énfasis en definir relaciones entre dos magnitudes a través de una regla de correspondencia, expresada algebraicamente, para encontrar y a partir de un valor de x; sin embargo, no se promueve el estudio de relaciones de covariación entre dichas magnitudes. Una revisión del currículo mexicano de educación secundaria permite constatar que se presenta una situación similar con respecto al estudio de la variación lineal; no se favorece el estudio de la variación de cada magnitud, ni de su variación conjunta. Más aún, no se promueve explícitamente el desarrollo del pensamiento variacional de los estudiantes (Bojórquez, Castillo y Jiménez, 2016), que es central para comprender adecuadamente los fenómenos de variación y cómo las nociones de función, derivada e integral permiten abordar su estudio en los cursos de Cálculo universitarios. Jiménez-Rodríguez (comunicación personal, 2018) plantea que en la escuela secundaria es donde debería darse el estudio de la variación, iniciando con el análisis del comportamiento variacional de una sola magnitud variable, previo al estudio de representaciones que requieren de la comprensión de la variación conjunta de dos variables, como las gráficas cartesianas. Además, considera que este estudio debe realizarse de manera integral con el tratamiento de números y cantidades en la recta numérica; sin embargo, el currículo no lo promueve explícitamente. Hacerlo de esta manera, permitiría reconocer que la variación lineal es una 
noción ligada a los procesos de cambio, en donde participan magnitudes variables, cada una de las cuales varía de manera uniforme con respecto al tiempo. Es importante mencionar que estas ideas fueron posteriormente publicadas Jiménez-Rodríguez (2020). Por otro lado, al elegir dos de estas magnitudes, se puede determinar que estas covarían de manera proporcional; es decir, al calcular la variación de una magnitud y compararla con la variación correspondiente de la otra, se puede establecer una relación de proporcionalidad entre las variaciones (Grijalva, comunicación personal, 2018).

Considerando que el currículo y libros de texto son los materiales principales de un profesor de matemáticas de secundaria, y si se toma en cuenta que en estos se plantea un estudio estático de la variación lineal que descuida sus propiedades esenciales, se pone de manifiesto la importancia de elaborar propuestas didácticas que provean a los profesores de matemáticas de una perspectiva más amplia sobre la enseñanza de la variación lineal, de manera que su práctica docente no se restrinja a presentar el enfoque limitado de los programas de estudio y libros de texto.

Es particularmente importante la formulación de propuestas didácticas que permitan intervenir en la etapa formativa de los profesores de matemáticas, de manera que favorezcan el desarrollo temprano de nuevos conocimientos y competencias para implementar y valorar procesos de instrucción más ricos y significativos para los estudiantes. Como lo expresan Godino, Giacomone, Batanero y Font (2017): "la formación didáctica de los profesores es un campo de investigación científica y tecnológica que reclama atención por parte de la Didáctica de la Matemática, pues el desarrollo del pensamiento y de las competencias matemáticas básicas de los alumnos depende, de manera esencial, de dicha formación.” (p. 91).

Aunque uno de los focos de atención en la investigación en Didáctica de las Matemáticas ha estado puesto en el profesor de matemáticas, es importante destacar que son pocas las investigaciones sobre el estudio que se hace de la variación en programas de formación de profesores, y más escasas son aún las propuestas formativas encaminadas a fortalecer los Conocimientos y Competencias Didáctico-Matemáticos de los futuros profesores sobre variación.

Por otro lado, aunque son pocas las investigaciones que reportan dificultades del profesorado en formación con respecto a la variación, hay investigaciones que reportan dificultades que profesores o futuros profesores de matemáticas presentan con relación a la proporcionalidad y la función, nociones estrechamente ligadas a la variación lineal. Por ejemplo, Wilhelmi, Godino y Lasa (2014) han reportado la existencia de conflictos que tienen futuros profesores entre el significado de función y de ecuación; Amaya, Pino-Fan y Medina (2016) destacan las limitaciones de futuros profesores para identificar los elementos de la función y relacionarlos en uno o varios registros de representación, así como para realizar transformaciones a las representaciones de la función. Con respecto a la proporcionalidad, Balderas, Block y Guerra (2014) encontraron deficiencias conceptuales en profesores de matemáticas que les dificultaron identificar propiedades de las relaciones de proporcionalidad y resolver problemas de escalas sucesivas y de relación aditiva.

A partir de estos estudios, se puede considerar que es importante acompañar con diseños didácticos el estudio de la variación lineal en la formación inicial de los profesores de matemáticas, de manera que se pueda abonar al desarrollo de conocimientos más amplios o más profundos en torno a esta noción, como lo pretenden los nuevos Planes de Estudio para las Escuelas Normales en México, en particular el Plan de estudios de la Licenciatura en Enseñanza y Aprendizaje en Educación Secundaria (SEP, 2018). 
Esta problemática, que se ha descrito a grandes rasgos, motivó el diseño de las actividades didácticas de Herrera (2020), que inició con la pretensión de promover un significado más rico sobre la variación lineal con profesores en formación inicial, pero que terminó ampliando sus propósitos para incluir el desarrollo de Conocimientos y Competencias Didáctico-Matemáticos sobre variación lineal.

\section{Herramientas teóricas inicialmente consideradas para el diseño de las actividades}

El Enfoque Ontosemiótico es una perspectiva teórica de la Didáctica de las Matemáticas desarrollada por Godino y otros investigadores (Godino, Batanero y Font, 2007) que integra de manera sistémica elementos importantes de diversas aproximaciones teóricas que han surgido en esta disciplina. A través de sus constructos teóricos, el EOS permite analizar y describir detalladamente diversos aspectos de los procesos de instrucción matemática, por ejemplo, la actividad matemática desarrollada por un estudiante, o bien los conocimientos y competencias que un profesor posee para la enseñanza de las matemáticas, y proponer acciones que lleven a su mejora progresiva.

En el EOS se toman como punto de partida dos elementos centrales: las situaciones problema y las prácticas matemáticas realizadas para resolverlas. A partir de estas dos nociones, se introducen las nociones de significado y objeto matemático primario. Se considera que una práctica matemática es toda acción o expresión realizada con la intención de resolver un problema, comunicar la solución a otros, validarla o generalizarla (Godino y Batanero, 1994).

En la enseñanza y el aprendizaje de las matemáticas, más que prácticas aisladas, lo que interesa es el desarrollo de sistemas de prácticas que lleven a la solución de problemas de un mismo tipo y que favorezcan la emergencia de objetos matemáticos. Y son estos sistemas de prácticas los que conforman el significado de los objetos que emergen de ellos. El sistema de prácticas en torno a la resolución de situaciones problema puede ser realizado por un individuo, o bien puede ser compartido o promovido por sujetos en una institución (o grupo), por lo cual, se considera necesario distinguir el significado personal (sistema de prácticas realizadas por un individuo) del significado institucional (sistema de prácticas compartido en una institución) que se tiene de un objeto matemático (Godino y Batanero, 1994).

Particularmente, en el diseño de actividades didácticas dentro de un sistema educativo toma relevancia el significado institucional de un objeto matemático, pues los procesos de instrucción matemática pretenden que los significados personales se acerquen cada vez más a los significados que institucionalmente se consideran apropiados, según el nivel educativo. En el EOS se definen distintos tipos de significados institucionales: de referencia, pretendido, implementado y evaluado (Godino, et al., 2007). Dado que este escrito se enfoca en el diseño de actividades didácticas, no en su implementación ni en su valoración, se caracterizarán únicamente el significado institucional pretendido (que corresponde al sistema de prácticas matemáticas que será incluido en la planeación de un diseño de instrucción) y el significado institucional de referencia (el sistema de prácticas matemáticas que se toma como referencia para determinar el significado institucional pretendido).

Cuando se aborda la resolución de una situación problema, se ponen en juego una serie de entidades matemáticas básicas que intervienen y emergen de las prácticas matemáticas realizadas, interactuando entre sí como una red de conocimiento. Estas entidades, denominadas objetos matemáticos primarios, permiten 
describir y analizar con detalle la actividad matemática llevada a cabo al intentar dar solución a la situación planteada. Se proponen en el EOS seis tipos de objetos matemáticos primarios (Godino, et al., 2007):

- Situaciones problema: aplicaciones extra matemáticas, ejercicios, tareas, problemas abiertos, etc.

- Lenguajes: términos, expresiones, notaciones, dibujos, gestos, expresados en diferentes registros (escrito, oral o gestual).

- Conceptos-definiciones: conceptos introducidos mediante definiciones o descripciones.

- Proposiciones-propiedades: enunciados sobre los conceptos.

- Procedimientos: algoritmos, operaciones, técnicas de cálculo, etc.

- Argumentos: enunciados para validar o explicar proposiciones y procedimientos.

Las nociones de práctica, significado institucional y objetos matemáticos primarios fueron las primeras herramientas teóricas del EOS que jugaron un papel en el diseño de las actividades didácticas de Herrera (2020), particularmente en la primera de ellas, que marcó la pauta para el diseño de las siguientes. Es por ello por lo que a continuación se describe cómo estas nociones teóricas dieron forma al significado pretendido de la variación lineal dentro del diseño, conjugando un enfoque variacional con el contenido propuesto en planes y programas de estudio y libros de texto. Posteriormente se presentará a detalle la Actividad 1 descrita con las herramientas teóricas del EOS.

\section{Proceso de diseño de la primera actividad didáctica: Significados institucionales}

La determinación de los significados institucionales, de referencia y pretendido, constituyeron el primer paso en el diseño de las actividades didácticas. Para ello, se realizó un análisis de prácticas matemáticas y objetos matemáticos primarios puestos de manifiesto en los planes y programas de estudio de secundaria. Se revisaron el Plan de estudios 2011 (SEP, 2011) y Aprendizajes Clave del Nuevo Modelo Educativo (SEP, 2017), así como libros de texto acordes a ellos (Figueras, Filloy, Ojeda, Rojano y Zubieta, 2013; Block, García y Balbuena, 2018).

En términos generales, se encontró que los documentos analizados plantean el siguiente sistema de prácticas (significado institucional pretendido por el currículo) para el estudio de la variación lineal. Se inicia con situaciones de variación lineal de contextos extra matemáticos donde existe una relación de proporcionalidad entre las magnitudes involucradas, mayormente planteadas en un lenguaje numérico en formato de tabla de valores. A partir de los datos de la tabla, se establecen propiedades de proporcionalidad entre las magnitudes que se emplean para encontrar valores faltantes, mediante el reconocimiento de la constante de proporcionalidad o usando la regla de 3. Con base en los valores de la tabla se establece una relación de correspondencia entre los valores de las magnitudes, la cual se representa algebraicamente de la forma $y=$ $k x$. Tomando los valores de las tablas numéricas, también se propone graficar puntos en el plano cartesiano para concluir que la gráfica de la relación de proporcionalidad es una línea recta que pasa por el origen. Posteriormente, a través de tablas numéricas se introducen situaciones problema más generales, también en contextos extra matemáticos, en las cuales ya no se tiene una relación de proporcionalidad entre las magnitudes involucradas. Sin embargo, en estas situaciones ya no se discute sobre relaciones de proporcionalidad, sino que el propósito es obtener una expresión algebraica de la forma $y=m x+b$ y la 
representación gráfica como línea recta, para luego establecer relaciones entre elementos característicos de ambas representaciones (ordenada al origen, pendiente, etc.).

Este sistema de prácticas propuesto para el estudio de la variación lineal en el currículo no explota la naturaleza variacional de las situaciones problema planteadas ni la relación que siempre existe entre las nociones de proporcionalidad y variación lineal. Es por ello por lo que en el trabajo de Herrera (2020) esos sistemas de prácticas se tomaron solamente como una parte del significado institucional de referencia para determinar el significado institucional pretendido por el diseño de las actividades didácticas. Con base en la literatura de investigación en matemática educativa, se optó por ampliar los sistemas de prácticas de referencia, considerando situaciones problema y objetos primarios propios de un tratamiento variacional, retomando los planteamientos de Panorkou y Malooney (2016) y de Jiménez (comunicación personal, 2018), en conjunción con las herramientas teóricas del EOS. Se inició la búsqueda de situaciones problema que favorecieran el desarrollo de sistemas de prácticas de los cuales emergiera la variación lineal como objeto matemático. El tipo de situaciones problema debía proveer un contexto de cambio, donde participaran magnitudes variables (de variación continua). De manera que, al elegir dos de estas magnitudes, se pudiera determinar si estas covarían o no de manera lineal, es decir, si las variaciones correspondientes de las magnitudes elegidas son directamente proporcionales.

Entonces, el significado institucional pretendido por el diseño, es decir, el sistema de prácticas a desarrollar en las actividades didácticas, para abordar las situaciones problema, debía partir de la identificación de las magnitudes variables involucradas en la situación planteada, el estudio de la manera como cada una de éstas varía y la búsqueda de relaciones de proporcionalidad entre las variaciones correspondientes de cada una de las magnitudes variables. El reconocimiento de esta propiedad da pie a su uso como argumento básico para decidir si se tiene o no un caso de variación lineal entre las magnitudes involucradas en la situación problema planteada. Además, se busca la caracterización gráfica y algebraica de la variación lineal, como una línea recta y una expresión de la forma $y=m x+b$, respectivamente.

De manera específica, se eligieron los siguientes objetos primarios como componentes del significado de variación lineal pretendido por el diseño:

- Lenguajes: Se seleccionaron los lenguajes planteados en el currículo de secundaria y libros de texto analizados. Por ello, se incluyeron representaciones numéricas/tabulares, gráficas cartesianas y expresiones algebraicas. Sin embargo, considerando las ideas de Jiménez (comunicación personal, 2018), se incluyó también la representación de magnitudes variables en rectas numéricas de una manera dinámica con la mediación de applets de GeoGebra. Se contempló el uso de términos y notaciones que indicaran la variación de las magnitudes, como: cambio, variación, constante, magnitud variable, incremento, flechas y otros símbolos para representar la variación, etc.

- Conceptos-definición: Dado que en el currículo y libros de texto analizados no se proporcionó una definición de la variación lineal, con el diseño se buscó favorecer la emergencia del siguiente concepto de variación lineal: es un tipo de variación conjunta entre dos magnitudes que varían de manera uniforme, de manera que existe una relación de proporcionalidad entre las variaciones correspondientes de las magnitudes variables. Esto involucró también al concepto de magnitud variable. Otros conceptos que se consideraron intervinientes en el diseño y por lo tanto no fueron definidos fueron el de variación, recta, pendiente, relación de proporcionalidad y constante de proporcionalidad. 
- Procedimientos: Cálculo de las variaciones de magnitudes variables a partir de valores numéricos de ambas magnitudes, cálculos de valores faltantes de las variaciones de las magnitudes mediante regla de tres, comparación de las variaciones de una magnitud considerando variaciones uniformes de la otra magnitud, cálculo de la constante de proporcionalidad entre las variaciones de ambas magnitudes mediante un cociente, obtención de la expresión algebraica de la relación entre las magnitudes variables, graficación de puntos en el plano cartesiano con los valores correspondientes de las magnitudes variables y cálculo de la pendiente de la recta que pasa por dos puntos mediante la fórmula $\frac{y_{2}-y_{1}}{x_{2}-x_{1}}$.

- Proposiciones-propiedades: si dos magnitudes varían de manera uniforme, su variación conjunta es variación lineal; las variaciones correspondientes de cada magnitud variable son directamente proporcionales; la variación lineal entre dos magnitudes variables se representa gráficamente como una línea recta y algebraicamente como una expresión de la forma $y=m x+b$; la constante de proporcionalidad entre las variaciones de las magnitudes variables es el mismo valor que la pendiente de la recta y que la constante $m$ de la expresión algebraica; en casos de variación lineal, si una magnitud aumenta la otra aumenta o disminuye de manera proporcional; en casos de variación lineal, no siempre hay una relación de proporcionalidad entre las magnitudes mismas, pero siempre la hay entre las variaciones correspondientes de cada una.

- Argumentos: se promueve el uso de las proposiciones-propiedades y procedimientos enlistados para argumentar si en la situación problema se tiene, o no, un caso de variación lineal entre dos magnitudes variables.

\section{Diseño de la primera actividad didáctica}

La Actividad 1, titulada "La edad canina", incluye todas las formas de lenguaje consideradas en el significado institucional pretendido por el diseño; sin embargo, se enfatiza el trabajo en las rectas numéricas, en lenguaje numérico y tabular. Parte de una situación problema planteada en lenguaje natural que favorece el tránsito hacia la representación de magnitudes variables en rectas numéricas para establecer una relación (de proporcionalidad) entre las variaciones de dichas magnitudes. Posteriormente, se traza una gráfica cartesiana. La propiedad de proporcionalidad entre las variaciones de las magnitudes se toma como base para llenar una tabla de valores faltantes. Finalmente, se obtiene una expresión algebraica para la relación entre las magnitudes, donde se puede asociar el coeficiente de la variable independiente con la constante de proporcionalidad entre las variaciones de las magnitudes. A continuación, se describe con detalle esta trayectoria.

Como punto de partida, se plantea en lengua natural el método propuesto por una veterinaria para calcular la edad biológica de los perros: Un perro de 1 año tiene la edad biológica de un ser humano de 20 años, y a partir de ese momento la edad biológica del perro aumenta 4 años por cada año que transcurre. Como se puede observar, el método establece una relación entre la variación de dos magnitudes variables (el tiempo y la edad biológica); ésta fue una característica que se consideró importante para elegirlo como base para la situación problema. A partir de este método, se formuló la situación problema de calcular la edad biológica de un perro correspondiente a cierta cantidad de años vividos, y viceversa, así como calcular cuánto varía una magnitud según la variación de la otra. 
La resolución de esta situación problema, a lo largo de la actividad, está guiada por tareas y preguntas que buscan la emergencia de diversos objetos matemáticos. En la Tabla 1 se presentan las primeras preguntas y tareas planteadas en torno al método para calcular la edad biológica de un perro, las cuales promueven como prácticas matemáticas la identificación de magnitudes variables y su representación en una recta numérica, así como la descripción de la manera como éstas varían y la identificación de una relación de proporcionalidad entre tales variaciones. En el desarrollo de estas prácticas se busca favorecer la emergencia de objetos como magnitud variable y variación, términos para describir la manera como las magnitudes cambian, lenguaje gráfico y lengua natural, propiedades referentes a que cada magnitud varía de manera uniforme y a que existe una relación de proporcionalidad entre la variación de una magnitud con respecto a la otra.

Tabla 1. Extracto \#1 de la Actividad Didáctica 1.

Los perros envejecen más rápido que los seres humanos, por ejemplo, un perro de seis años es considerado un perro adulto por la madurez y el envejecimiento (edad biológica) que ha alcanzado su cuerpo. Existen diferentes métodos para estimar la edad biológica de un perro, la veterinaria Mirtha Smith propone la siguiente técnica (adaptada de Smith, 2017):

Un perro de 1 año tiene la edad biológica de un ser humano de 20 años, y a partir de ese momento la edad biológica del perro aumenta 4 años por cada año que transcurre

1. En la situación planteada, ¿qué magnitudes cambian conforme pasa el tiempo?

2. Representa en la siguiente recta numérica el cambio de la edad del perro conforme pasan los años.

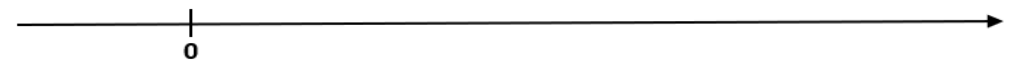

a) Describe cómo es el cambio de la edad del perro conforme pasa el tiempo.

3. Representa en la siguiente recta numérica el cambio de la edad biológica del perro conforme pasan los años.

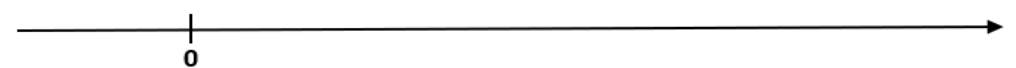

b) Describe cómo es el cambio de la edad biológica del perro conforme pasa el tiempo.

c) ¿Qué relaciones encuentras entre el cambio de ambas magnitudes (edad del perro y edad biológica)?

Posteriormente, se plantea el trabajo con rectas numéricas dinámicas (Figura 1), para lo cual se diseñó un applet (disponible en https://ggbm.at/ufgxxfxk) que presenta por separado cada magnitud variable como un segmento dirigido sobre la recta numérica, cuya magnitud aumenta de manera continua. Para facilitar la emergencia de la propiedad que tienen las magnitudes de variar de manera uniforme, y para representar de manera dinámica la relación establecida por el método de la veterinaria, se muestra un rastro uniforme de puntos correspondientes a valores de la magnitud, que ésta va dejando cada cierto periodo de tiempo. Se muestra primero la edad del perro (Figura 1a) variando uniformemente y dejando rastro por cada año transcurrido. Después, se muestra también edad biológica (Figura 1b) variando de manera uniforme, pero dejando rastro cada cuatro años, de manera que se pueda observar la variación conjunta de ambas y se puedan establecer relaciones entre ellas y entre sus variaciones. 


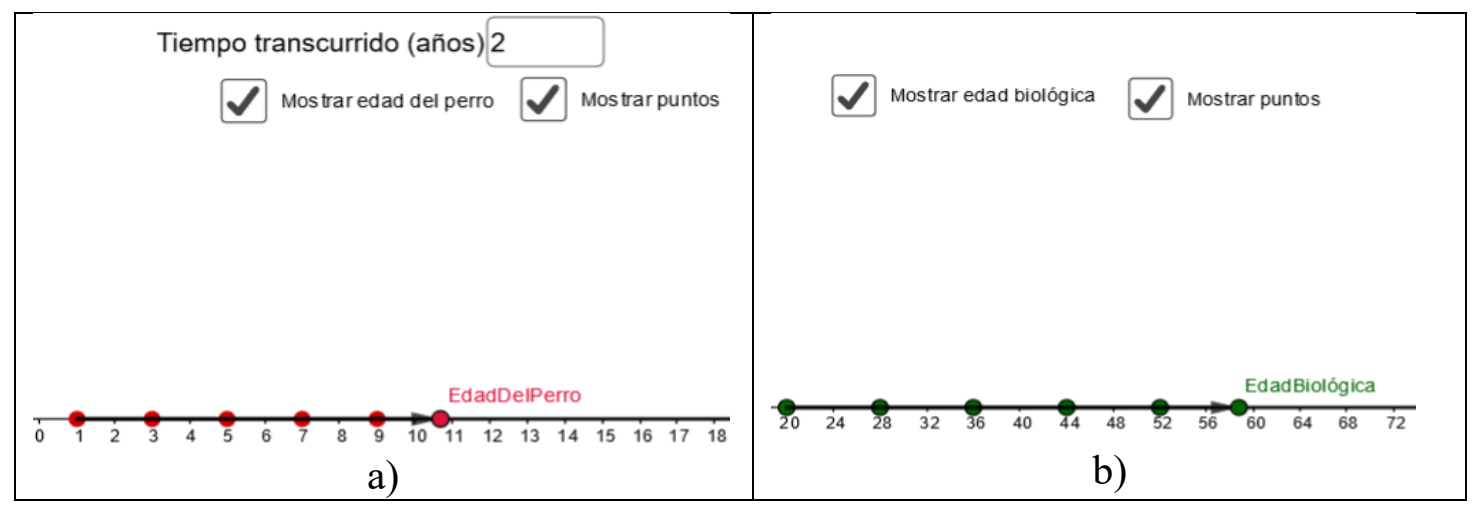

Figura 1. Variación uniforme de magnitudes variables en la recta numérica

El applet de la Figura 1 permite cambiar cada cuántos años se muestra el rastro de puntos correspondiente a la edad del perro, y observar que esto modifica el rastro de puntos correspondiente a la edad biológica. Con estas características del applet, y guiando su exploración, se pretende favorecer la emergencia de las siguientes propiedades: las magnitudes varían de manera uniforme, constante y, además, se tiene una relación de proporcionalidad entre las variaciones de las magnitudes, pues cada que la edad del perro varía cierta cantidad de años, la edad biológica varía cuatro veces dicha cantidad.

Después de la exploración del applet se plantea un conjunto de preguntas (Tabla 2) orientadas a reforzar la emergencia de las dos propiedades descritas arriba sobre las variaciones de las magnitudes.

Tabla 2. Extracto \#2 de la Actividad 1.

1. Con el uso del applet, ¿observaste algo distinto sobre el cambio de cada magnitud con respecto
a la representación gráfica que realizaste? Si es así, descríbelo.
2. ¿Qué relaciones encuentras entre la edad del perro y su edad biológica?
3. Crea una gráfica cartesiana donde representes la relación que encuentras entre ambas edades.
4. Según los datos proporcionados por la veterinaria:
a) ¿cuánto aumenta la edad biológica cada dos años?
b) ¿Cuánto aumenta la edad biológica cada tres años?
c) ¿Cuánto aumenta la edad biológica cada seis años?
5. Con los datos proporcionados por la veterinaria ¿podrías decidir cuánto aumenta la edad
biológica del perro cada 7 años? Si es así, describe el procedimiento que empleaste.

También, se solicita el trazado de una gráfica para empezar a favorecer la emergencia de la propiedad que tiene la variación lineal de ser representada por una línea recta (cuando las magnitudes varían de manera continua). Se promueve que los futuros profesores realicen cálculos aritméticos para obtener variaciones de la edad biológica según la variación de la edad del perro y con base en las variaciones visualizadas en el applet.

Posteriormente (Tabla 3), se solicita llenar una tabla de valores faltantes. Sabiendo el valor de una magnitud, se pide encontrar el valor de la otra, con base en el cálculo de la variación de cada magnitud y la relación de proporcionalidad entre dichas variaciones. En esta parte se espera que intervengan procedimientos como la regla de tres con valores de las variaciones o incrementos de las magnitudes. La columna central, está orientada a que los futuros profesores establezcan un procedimiento que relacione una magnitud con otra, con el propósito de favorecer la emergencia de una expresión algebraica que relacione ambas magnitudes variables. 
Tabla 3. Extracto \#3 de la Actividad 1.

En equipo llenen la siguiente tabla con la técnica propuesta por la veterinaria Mirtha Smith y encuentren una fórmula o expresión algebraica que les permita calcular la edad biológica del perro a partir de la edad que tiene

\begin{tabular}{|c|c|c|c|c|}
\hline $\begin{array}{c}\text { Incrementos } \\
\text { edad del } \\
\text { perro }\end{array}$ & Edad del perro & $\begin{array}{c}\text { Regla/ } \\
\text { Procedimiento }\end{array}$ & $\begin{array}{c}\text { Edad } \\
\text { biológica } \\
\text { del perro }\end{array}$ & $\begin{array}{c}\text { Incrementos } \\
\text { edad } \\
\text { biológica del } \\
\text { perro }\end{array}$ \\
\hline & & & 20 & \\
\hline & & & 24 & \\
\hline & 3 & & & \\
\hline & & & 36 & \\
\hline & 7 & & & \\
\hline & & & 64 & \\
\hline & & & 84 & \\
\hline & 17 y 6 meses & & & \\
\hline & 17 y 9 meses & & & \\
\hline & $\begin{array}{c}18 \text { años y } 6 \\
\text { meses }\end{array}$ & & & \\
\hline & & & 92 & \\
\hline & $\begin{array}{c}19 \text { años y } 6 \\
\text { mes }\end{array}$ & & & \\
\hline & 20 años & & & \\
\hline
\end{tabular}

1. ¿Qué relación encuentras entre los incrementos de la edad del perro y los incrementos de su edad biológica?

2. ¿Encuentras relaciones de proporcionalidad en los datos de la tabla? Si es así, menciona en cuáles y cuál es la constante de proporcionalidad.

3. Explica los procedimientos que utilizaste para llenar las filas en donde la edad del perro está dada en años y meses.

4. ¿Encontraste una expresión algebraica que funcione para todos los datos de la edad del perro y su edad biológica? Sí es así, descríbela.

Finalmente, en la Actividad 1 se presenta un método distinto para calcular la edad biológica de perros de razas de distinto tamaño. Se solicita a los futuros profesores elaborar las expresiones algebraicas para calcular la edad biológica en cada raza. Se plantean preguntas para promover la emergencia de la propiedad de que la constante de proporcionalidad entre las variaciones de las magnitudes en el método de cada raza corresponde a cierto coeficiente de las expresiones algebraicas, las cuales tienen la forma $y=m x+b$, como se muestra en la Tabla 4.

Tabla 4. Extracto \#4 de la Actividad 1.

1. Otros veterinarios como Stanley Coren (2018) menciona que existen diversos factores que deben ser tomados en cuenta para calcular la edad de nuestras mascotas, como su tamaño o raza. El especialista calcula la edad biológica de los perros de la siguiente manera: 
En todos los perros el primer año de cachorro corresponde a los primeros 16 años de un ser humano. A partir de este momento por cada año que transcurre, las razas pequeñas envejecen 4 años, mientras que las razas medianas envejecen 6 y las razas grandes 7 .

a) Determina una expresión algebraica que funcione para calcular la edad biológica de cada uno de los perros (raza pequeña, raza mediana y raza grande).

b) ¿De los datos dados por el veterinario cuáles aparecen directamente en la expresión algebraica que modela cada método? ¿Qué papel tienen en la expresión algebraica estos datos?

La Actividad 1 se diseñó como el primer paso a dar para la emergencia de diversos objetos matemáticos primarios involucrados en las prácticas que se busca que los futuros profesores adopten como su sistema de prácticas matemáticas al trabajar con todas las actividades de la propuesta. Sin embargo, se percibió la falta de elementos de reflexión, en la Actividad 1, que fueran de utilidad para la práctica docente de los futuros profesores, más allá del enriquecimiento de sus conocimientos matemáticos.

Volviendo a ver la problemática, se puso el foco de atención no sólo en la importancia y necesidad del desarrollo de un conocimiento matemático más rico en la formación inicial de profesores, sino también en el desarrollo de otro tipo de conocimientos y competencias necesarias para llevar a cabo una buena práctica docente en el aula. Según Llinares (2007), diversas investigaciones destacan que la formación inicial de los profesores de matemáticas debe tomar en consideración las 'tareas profesionales' que se espera que los futuros profesores realicen en el aula, es decir, formar al profesor a partir de la práctica de enseñar matemáticas. Esto derivó en la tarea de ampliar el diseño inicial de la Actividad 1 para integrar nuevos elementos de utilidad para la práctica docente de los futuros profesores, y el EOS tendría un papel muy importante en esta nueva tarea.

\section{Modelo de Conocimientos y Competencias Didáctico-Matemáticos del profesor de matemáticas}

Dentro del grupo de investigación del EOS se han realizado distintos desarrollos teóricos centrados en el profesor de matemáticas, para atender la necesidad de contar con un modelo para evaluar los conocimientos didáctico-matemáticos del profesor y desarrollar sus competencias didáctico-matemáticas, de manera que se pueda orientar la formación de profesores de matemáticas para su mejora.

El modelo de Conocimientos y Competencias Didáctico-Matemáticos del profesor de matemáticas (modelo CCDM) (Godino, Giacomone, Batanero y Font, 2017.) considera que los profesores de matemáticas deben poseer dos tipos de conocimiento, conocimiento matemático (común y ampliado) y conocimiento especializado en torno a la enseñanza de las matemáticas (Pino-Fan y Godino, 2015), es decir, conocimiento didáctico-matemático. Este segundo, se refiere al conocimiento de las distintas facetas que están presentes en todo proceso de instrucción matemática: Epistémica, cognitiva, afectiva, interaccional, mediacional y ecológica (Godino et al., 2017):

- Faceta epistémica: Es el conocimiento especializado sobre el contenido matemático, sobre la diversidad de significados y sus conexiones, sobre la variedad de objetos matemáticos primarios puestos en juego en la actividad matemática relacionada a tal contenido (lenguajes, proposiciones, conceptos, argumentos, situaciones problema, procedimientos). Es decir, es la forma en que el profesor comprende y conoce las matemáticas. 
- Faceta cognitiva: Corresponde al conocimiento de cómo los estudiantes aprenden matemáticas y cómo evoluciona su aprendizaje.

- Faceta afectiva: Conocimientos sobre aspectos afectivos del estudiante, sobre sus emociones, actitudes, motivaciones y creencias de los estudiantes con respecto a las matemáticas.

- Faceta interaccional: Referente al conocimiento sobre las interacciones que se pueden establecer en el aula.

- Faceta mediacional: Conocimiento de los recursos tecnológicos, materiales y temporales que son adecuados para favorecer el aprendizaje de los estudiantes.

- Faceta ecológica: Conocimientos con relación a aspectos curriculares, sociales, contextuales, políticos y económicos que condicionan todo proceso de instrucción.

Por otro lado, en el CCDM se considera que el profesor de matemáticas, además de poseer una competencia matemática que le permita resolver problemas matemáticos, debe desarrollar una competencia más general para poder hacer frente a los problemas didácticos que se presenten en el aula de matemáticas. Es decir, el profesor de matemáticas debe desarrollar la competencia de análisis e intervención didáctica, que le permita diseñar e implementar actividades de aprendizaje, así como valorar su pertinencia mediante un análisis didáctico, de manera que pueda refinar su propuesta inicial e iniciar un nuevo ciclo de implementación y valoración que gradualmente lo lleve a la mejora de su práctica docente (Breda, Pino-Fan y Font, 2017).

La competencia de análisis e intervención didáctica se puede dividir en cinco subcompetencias (Godino et al., 2017),

- Competencia de análisis de significados globales (identificación de situaciones problema y sistemas de prácticas implicadas en su resolución);

- Competencia de análisis ontosemiótico de las prácticas (identificación de objetos matemáticos primarios y procesos que juegan un papel en las prácticas realizadas);

- Competencia de gestión de configuraciones y trayectorias didácticas (identificación de la secuencia temporal de interacciones entre profesor, estudiante, contenido y recursos mediacionales);

- Competencia de análisis normativo (reconocimiento de normas y meta-normas que condicionan y soportan el proceso de instrucción);

- Competencia de análisis de la idoneidad didáctica (valoración del proceso de instrucción para formular posibles mejoras).

Dentro de esta perspectiva teórica ampliada que proporciona el CCDM al seno del EOS, en el trabajo de Herrera (2020) se retomó la faceta epistémica del conocimiento didáctico-matemático del profesor, al considerar que ésta tiene relación cercana con los planteamientos del diseño inicial de la Actividad 1, orientada al desarrollo de conocimientos matemáticos sobre variación lineal.

Con respecto a la competencia de análisis e intervención didáctica, de las cinco subcompetencias didácticomatemáticas, el trabajo de Herrera (2020) eligió la competencia de análisis ontosemiótico de las prácticas. Se tomó como supuesto que al analizar la actividad matemática realizada al resolver problemas (identificando los objetos matemáticos involucrados en ella) se podrían reforzar los significados desarrollados, al iniciar a los futuros profesores en un proceso de toma de consciencia y reflexión sobre qué conceptos, propiedades, etc., ponen en juego en sus propias prácticas matemáticas. Es decir, se consideró que este tipo de análisis podría despertar en los profesores una sensibilidad sobre la complejidad de las prácticas matemáticas y la diversidad de objetos matemáticos primarios implicados en ellas. Por otro lado, 
la consideración de esta subcompetencia llevó a retomar como elemento importante en el diseño a la faceta cognitiva, además de la epistémica, pues el análisis de prácticas matemáticas propias y objetos matemáticos primarios podría ser una herramienta útil para el análisis y retroalimentación de las prácticas de un estudiante.

\section{Ampliación de la actividad 1 para incluir el desarrollo de Conocimientos y Competencias Didáctico-Matemáticos}

Tomando como base el diseño inicial de la Actividad 1 que buscaba promover el desarrollo de un significado más rico sobre variación lineal (con lo cual se atiende parte de la faceta epistémica del conocimiento didáctico-matemático), se prosiguió con la formulación de tareas y preguntas de reflexión que atendieran la faceta cognitiva y favorecieran el desarrollo de aspectos de la competencia de análisis ontosemiótico de las prácticas.

Se decidió iniciar la reflexión considerando el desarrollo de la competencia elegida, para ello, siguiendo la propuesta de Giacomone (2018), se formularon preguntas orientadas a establecer qué son un concepto, una propiedad y un procedimiento matemáticos, como se muestra en la Tabla 5. A partir de la caracterización de esos tres objetos matemáticos primarios, se propusieron tareas de análisis de las prácticas realizadas al resolver las tareas matemáticas planteadas en la Actividad 1. Estas preguntas y tareas de análisis se debían contestar de dos formas, primero desde una postura personal y también después desde un consenso logrado mediante una discusión grupal.

Tabla 5. Extracto \#5 de la Actividad 1.

1. ¿Qué es para ti un concepto matemático? Enlista los conceptos matemáticos que intervienen en la resolución de la actividad.

2. ¿Qué es para ti un procedimiento matemático? Describe los procedimientos matemáticos que te permitieron resolver la actividad.

3. ¿Qué es para ti una propiedad matemática? ¿Qué propiedades matemáticas identificas en la actividad que acabas de realizar?

Una vez realizado el análisis de las prácticas realizadas para identificar los objetos implicados en ellas, se plantearon tareas de análisis de la práctica matemática de un estudiante hipotético de secundaria, para atender la faceta cognitiva que trata sobre el conocimiento sobre el aprendizaje del estudiante. Para ello, se introdujo la respuesta de un estudiante a la situación problema planteada a los futuros profesores en la Actividad 1, en la cual el estudiante hacía uso de la regla de tres (Tabla 6). La tarea consistía en valorar la validez del procedimiento del estudiante y darle una retroalimentación. Con este tipo de tareas, se espera que el futuro profesor reflexione sobre su propia práctica matemática tomando como herramienta los tres objetos primarios elegidos, de manera que reflexione sobre qué conceptos, procedimientos y propiedades nuevos aprendió con lo realizado en la parte de trabajo matemático de la Actividad 1, cuáles de esos tres objetos primarios está poniendo en juego un estudiante al resolver un problema matemático y cómo puede orientarlo para mejorar su aprendizaje.

Tabla 6. Extracto \#6 de la Actividad 1.

1. Un estudiante de secundaria calculó la edad biológica de un perro de 15 años utilizando el método de la veterinaria Mirtha Smith como se muestra en la imagen: 


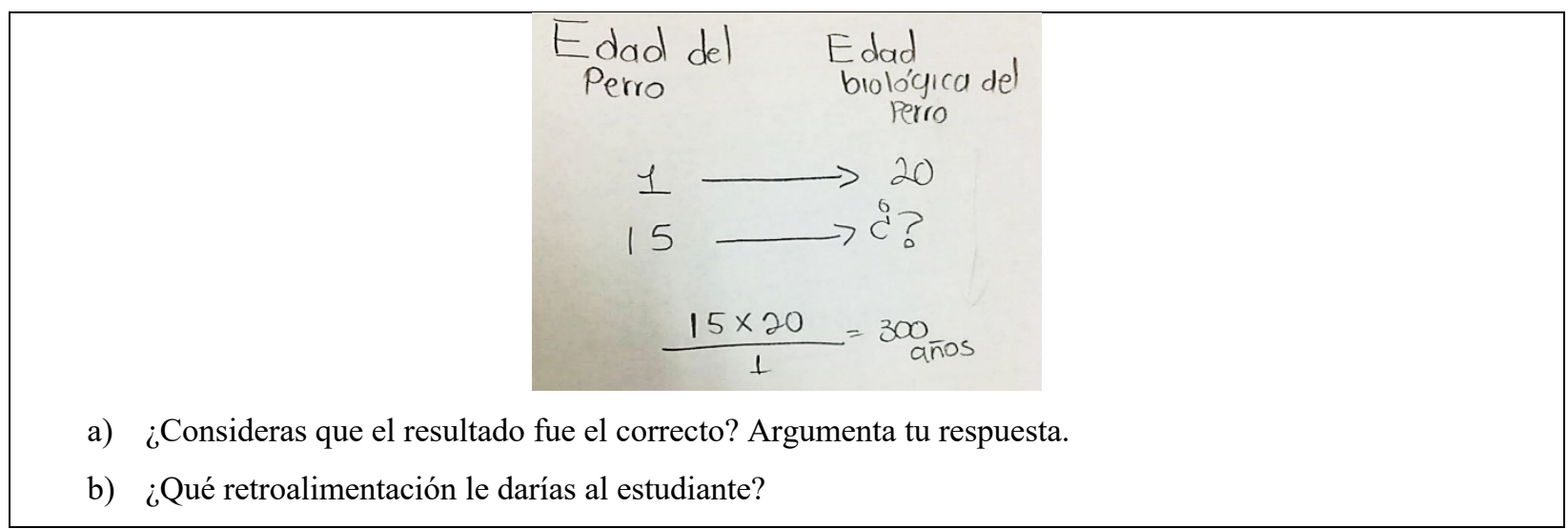

El diseño final de la actividad 1 concluyó al integrar estos dos tipos de tareas, enfocadas al análisis de las prácticas propias, y de las prácticas de un estudiante hipotético, para identificar tres de los seis objetos matemáticos primarios. Con ello, el diseño se reorientó hacia el desarrollo de aspectos importantes del conocimiento y competencias didáctico-matemáticos de los futuros profesores, y ya no solo de sus conocimientos matemáticos.

\section{Diseño final de las actividades didácticas}

El diseño final de la propuesta didáctica de Herrera (2020) incluyó cinco actividades, en las cuales se favorece el trabajo en diversas formas de lenguaje y el uso de applets de GeoGebra.

- Actividad 1, "La edad canina", que ya se describió a detalle.

- Actividad 2, "El peso y la estatura", en la cual se presenta un modelo lineal del peso ideal según la estatura y se contrasta con datos propuestos por el Instituto Mexicano del Seguro Social (IMSS). Se enfatiza el trabajo con gráficas cartesianas, la noción de pendiente de una recta y la comparación de un caso de variación lineal con uno que no es de variación lineal.

- Actividad 3, "Llenado de recipientes cilíndricos". En esta actividad se presenta un applet que modela el llenado de un recipiente cilíndrico. Se busca diferenciar dos tipos de variación lineal de la forma $y=m x+b$, cuando $b=0$ y cuando $b \neq 0$, para resaltar que la proporcionalidad entre las variaciones de las magnitudes es una propiedad inherente a la variación lineal, mientras que la proporcionalidad entre las magnitudes no.

- Actividad 4. "Vaciado de recipientes cilíndricos", en la cual se modela con un applet de GeoGebra el vaciado de un recipiente cilíndrico. En esta actividad se busca introducir la constante de proporcionalidad negativa, y distinguir la variación lineal de la variación inversamente proporcional, a la cual comúnmente se le asocia la frase "si una magnitud aumenta, la otra disminuye...".

- Actividad 5, "Una clase de matemáticas" trata sobre la confusión de una profesora entre la variación inversamente proporcional y variación lineal con constante negativa. Se busca reafirmar la caracterización de la variación lineal a partir del establecimiento de la proporcionalidad entre las variaciones correspondientes de las magnitudes variables involucradas.

Cada una de las cinco actividades se organizó en tres partes, para atender cada uno de los tres elementos que se pretende desarrollar en los profesores con respecto a la variación lineal: el conocimiento matemático, la competencia de identificación de objetos matemáticos primarios y el conocimiento didáctico-matemático. A continuación, se describe brevemente cada parte. 
La Parte I de las actividades se destinó al trabajo matemático. Se incluyeron situaciones problema de variación lineal y situaciones que no eran de variación lineal. Se consideraron diversos contextos como: calcular la edad biológica de un perro con respecto a los años vividos; calcular el peso $(\mathrm{kg})$ de una persona a partir de la estatura y comparar con los datos propuestos por el Instituto Mexicano de Seguro Social (IMSS); el llenado y vaciado de recipientes cilíndricos; y calcular las dimensiones de un rectángulo de área constante. Estas situaciones planteadas implicaban el uso de al menos dos formas de lenguaje: rectas numéricas dinámicas en GeoGebra, gráficas cartesianas, tablas de valores y expresiones algebraicas.

La Parte II de cada actividad didáctica se enfocó en la identificación de objetos matemáticos primarios en sus respuestas a la Parte I. En la primera actividad se buscó que los futuros profesores describieran lo que entienden por tres de los objetos matemáticos primarios, conceptos, procedimientos y propiedades, para posteriormente llegar a un consenso grupal. En las actividades posteriores, empleando la caracterización acordada grupalmente para los tres objetos primarios, se les solicitó a los futuros profesores que identificaran los objetos matemáticos primarios en sus propias prácticas matemáticas, que fueron desarrolladas en la Parte I de trabajo matemático.

Con respecto a la Parte III, orientada al análisis de respuestas dadas por estudiantes hipotéticos de secundaria, se presentó a los futuros profesores la respuesta de un supuesto estudiante de secundaria ante una tarea matemática relacionada con lo realizado en la Parte I, con el objetivo de que analizaran la actividad matemática manifestada en la respuesta del estudiante, usando los objetos matemáticos primarios identificados en la Parte II, y que con base en tal análisis crearan estrategias para retroalimentar al estudiante. Cabe mencionar que en cada una de estas tres partes se plantearon diferentes modalidades de trabajo: individual, en equipo de tres personas y discusión grupal, de manera que se favoreciera la emergencia de significados personales con el trabajo individual, su negociación durante las interacciones con los miembros del equipo, y consensos y su institucionalización tras discusiones grupales.

\section{Reflexiones finales}

En la literatura de Matemática Educativa es común encontrar artículos de investigación en los cuales los diseños, o materiales, elaborados para implementarse con los sujetos de estudio son una parte secundaria del contenido y, por ello, no son mostrados o no se describe a detalle su proceso de formulación. Por otro lado, en artículos de revista denominados comúnmente como "contribuciones a la docencia" o "experiencias de aula", aunque los materiales son más ampliamente descritos, su proceso de diseño también suele quedar oculto.

Considerando tal situación, en este escrito se decidió poner en el centro de discusión al proceso de diseño de las actividades didácticas de la tesis de maestría de Herrera (2020), y no su implementación, el análisis de los datos emanados del trabajo con los futuros profesores, ni la discusión de los resultados obtenidos. Otro aspecto que jugó un papel importante para esta decisión fue que la tesis de Herrera es producto de un programa de posgrado de orientación profesional, y no de investigación, por lo cual la parte central del trabajo de tesis fue la formulación de un diseño didáctico guiado por una perspectiva teórica de la Matemática Educativa para atender una problemática específica.

La pretensión de este artículo fue servir como base a estudiantes que inician su formación como matemáticos educativos, así como a profesores, para reflexionar sobre el proceso de diseño de materiales didácticos fundamentados teóricamente dentro de la Matemática Educativa, tomando como ejemplo el proceso seguido 
para formular las actividades didácticas de Herrera (2020). Es importante declarar que en este escrito no se está proponiendo una metodología de diseño didáctico. Más bien, se buscó resaltar que el proceso de diseño didáctico no es lineal ni sencillo, sino más bien un complejo ir y venir entre diferentes elementos que entran en juego: identificar una problemática educativa importante que pueda ser atendida, al menos en cierto grado, documentarse sobre la problemática con base en producción científica de Matemática Educativa, hacer un análisis crítico de planes y programas de estudio, seleccionar referentes teóricos que permitan identificar pautas y elementos centrales en la propuesta didáctica a diseñar, entre otros. Cada elemento va arrojando luz sobre los demás, favoreciendo su evolución conjunta.

Es importante destacar el papel central que tuvo el Enfoque Ontosemiótico. Por un lado, las nociones de sistema de prácticas y objetos matemáticos primarios permitieron realizar un análisis detallado de planes y programas de estudio, identificar prácticas y objetos que estaban ausentes y que valía la pena promover, según la literatura científica, así como determinar claramente el contenido matemático del diseño de las actividades, su organización y secuenciación. Por otro lado, el modelo de Conocimientos y Competencias Didáctico-Matemáticos, permitió conectar el trabajo matemático de los futuros profesores con tareas de reflexión sobre su conocimiento matemático y el de los estudiantes, que pudiera sembrar la semilla de una práctica docente distinta a la tradicional.

Los productos del trabajo de Herrera no fueron únicamente las cinco actividades descritas, sino que, a partir del proceso de reflexión llevado a cabo al realizar el diseño de éstas, se encontró que el tipo de lenguaje elegido en las tareas de cada actividad ponía cierto matiz a las prácticas matemáticas realizadas y a los objetos matemáticos que intervenían y emergían de ellas. Herrera (2020) planteó, entonces, cuatro distintos significados de variación lineal para reorganizar la descripción del significado institucional pretendido por el diseño: como representación analítica de la forma $y=m x+b$, como representación gráfica en rectas numéricas, como línea recta en el plano cartesiano y como tabla de valores con variaciones directamente proporcionales. Este es un ejemplo de cómo el proceso de diseño guiado por una perspectiva teórica puede dar paso a la producción de nuevo conocimiento y contribuciones teóricas.

Por otro lado, la problemática abordada y el trabajo implicado en el diseño de las actividades motivaron a Herrera a explorar más a fondo la relación de la variación lineal con los significados pragmáticos de la proporcionalidad de Godino, Beltrán-Pellicer, Burgos, y Giacomone, (2017) y los significados parciales de la función de Pino-Fan, Parra-Urrea y Castro (2019), identificando prácticas matemáticas específicas relacionadas con estas dos nociones que se promovían en los significados pretendidos en su diseño, de manera que esbozó con ellas una reformulación del significado institucional de referencia sobre variación lineal en su trabajo, mostrando que el proceso de diseño no es lineal.

\section{Agradecimientos}

Se hace un reconocimiento al Consejo Nacional de Ciencia y Tecnología (CONACYT) por la beca 862619 y a la Universidad de Sonora por el apoyo brindado para el diseño y realización de la propuesta formativa discutida en este trabajo.

\section{Referencias}

Amaya, T., Pino-Fan, L. y Medina, A. (2016). Evaluación del conocimiento de futuros profesores de matemáticas sobre las transformaciones de las representaciones de una función. Educación Matemática, 28(3), 111-144. 
Balderas, R., Block, D. y Guerra, M. (2014). Sé cómo se hace, pero no por qué”. Fortalezas y debilidades de los saberes sobre la proporcionalidad de maestros de secundaria. Educación Matemática, 26(2), 7-32.

Block. D., García, S. y Balbuena, H. (2018). Matemáticas 1. Secundaria. Conecta Más. Ciudad de México: D.R.SM de Ediciones. (p. 58-65).

Bojórquez A., Castillo, J. M. y Jiménez, J. R. (2016). Development of the variational thought in secondary students. Congreso Internacional en Tecnología y su Integración en la Educación Matemática (TIME). 29 de junio al 2 de julio de 2016. Austrian Center for Didactics of Computer Algebra (ACDCA) y Facultad de Ciencias de la UNAM. Ciudad de México. Disponible http://www.acdca.ac.at/fileadmin/Mathematik_Uploads/ACDCA/TIME2016/Garcia_ao.pdf

Breda, A., Pino-Fan, L. y Font, V. (2017). Meta didactic-mathematical knowledge of teachers: criteria for the reflections and assessment on teaching practice. Eurasia Journal of Mathematics, Science \& Technology Education, 13(6), 1893-1918

Dávila-Araiza, T. y Grijalva, A. (2020). Hacia una distinción didáctica entre el Cálculo y el Análisis. El caso de la noción de variable. En A.I. Sacristán, J.C. Cortés-Zavala \& P.M. Ruiz-Arias, (Eds.). Mathematics Education Across Cultures: Proceedings of the 42nd Meeting of the North American Chapter of theInternational Group for the Psychology of Mathematics Education, Mexico (pp. 1177-1181). Cinvestav /AMIUTEM / PME-NA. https:/doi.org/10.51272/pmena.42.2020

Figueras, O., Filloy, E., Ojeda, A., Rojano, M. y Zubieta, G. (2013). Matemáticas 2. Serie Aqua. Editorial Esfinge: México (p. 201-207).

Giacomone, B. (2018). Desarrollo de competencias y conocimientos didáctico-matemáticos de futuros profesores de educación secundaria en el marco del enfoque ontosemiótico (Tesis doctoral no publicada). Universidad de Granada, España. Disponible en: http://enfoqueontosemiotico.ugr.es/tesis/Tesis_Giacomone.pdf

Godino, J. D., Batanero, C. y Font, V. (2007). The onto-semiotic approach to research in mathematics education. ZDM. The International Journal on Mathematics Education, 39(1), 127-135.

Godino, J. D., Beltrán-Pellicer, P., Burgos, M. y Giacomone, B. (2017). Significados pragmáticos y configuraciones ontosemióticas en el estudio de la proporcionalidad. En J. M. Contreras, P. Arteaga, G. R. Cañadas, M. M. Gea, B. Giacomone, \& M. M. López-Martín (Eds.), Actas del Segundo Congreso International Virtual sobre el Enfoque Ontosemiótico del Conocimiento y la Instrucción Matemáticos. Disponible en, enfoqueontosemiotico.ugr.es/civeos.html

Godino, J. D., Giacomone, B., Batanero, C. y Font, V. (2017). Enfoque ontosemiótico de los conocimientos y competencias del profesor de matemáticas. Boletim de Educação Matemática, 31(57), 90-113

Godino, J. D., Giacomone, B., Batanero, C. y Font, V. (2017). Enfoque Ontosemiótico de los Conocimientos y Competencias del Profesor de Matemáticas. Boletim de Educação Matemática, 31(57), 90-113.

Godino, J. D. y Batanero, C. (1994). Significado institucional y personal de los objetos matemáticos. Recherches en Didactique des Mathématiques, 14(3), 325-355

Herrera, K. J. (2020). Propuesta formativa para el desarrollo de Conocimientos y Competencias DidácticoMatemáticos de futuros profesores de secundaria sobre variación lineal (Tesis de maestría no publicada). Universidad de Sonora, México.

Ímaz, C. y Moreno, L. (2010). La génesis y la enseñanza del cálculo. Las trampas del rigor. México: Trillas

Jiménez, J., Grijalva, A., Milner, F., Dávila-Araiza, M. T. y Romero, F. (en prensa). Reconceptualización didáctica del Cálculo.

Jiménez-Rodríguez, J. (2020). Level-zero covariational reasoning in secondary school mathematics. En A.I. Sacristán, J.C. Cortés-Zavala \& P.M. Ruiz-Arias, (Eds.). Mathematics Education Across Cultures: Proceedings of the 42nd Meeting of the North American Chapter of the International Group for the Psychology of Mathematics Education, Mexico (pp. 301-304). Cinvestav / AMIUTEM / PME-NA. https:/doi.org/10.51272/pmena.42.2020

Moreno-Armella, L. (2014). An essential tension in mathematics education. ZDM Mathematics Education, 46(4), 621633. doi: 10.1007/s11858-014-0580-4

Panorkou, N. y Maloney, A. P. (2016). Early Algebra: Expressing Covariation and Correspondence, Teaching Children Mathematics TCM, 23(2), 90-99. Recuperado de https://pubs.nctm.org/view/journals/tcm/23/2/articlep90.xml 
Pino-Fan, L. y Godino, J. D. (2015). Perspectiva ampliada del conocimiento didáctico-matemático del profesor. PARADIGMA, 36(1), 87-109.

Pino-Fan, L., Parra-Urrea, Y. y Castro, W. F. (2019). Significados de la función pretendidos por el currículo de matemáticas chileno. Magis, Revista Internacional de Investigación en Educación, 11(23), 201-220. doi: 10.11144/Javeriana.m11-23.sfpc

Secretaría de Educación Pública (2018). Plan de estudios 2018. Licenciatura en Enseñanza y Aprendizaje de las Matemáticas en Educación Secundaria. Disponible en https://www.cevie-dgespe.com/index.php/planes-deestudios-2018/120

Secretaría de Educación Pública. (2017). Aprendizajes Clave para la Educación Integral. Ciudad de México. SEP. Recuperado https://www.planyprogramasdestudio.sep.gob.mx/descargables/APRENDIZAJES CLAVE PARA LA EDU CACION_INTEGRAL.pdf

SEP. Secretaría de Educación Pública. (2011). Programa de estudios 2011 guía para el maestro, educación básica. Secundaria. Matemáticas. México, D.F: SEP. Recuperado de: https://www.gob.mx/cms/uploads/attachment/file/18394/Programa. Secundaria tercer_grado Matematicas g uia para maestros.pdf

Wilhelmi, M., Godino, J. D. y Lasa, A. (2014). Significados conflictivos de ecuación y función en estudiantes de profesorado de secundaria. En M. T. González, M. Codes, D. Arnau y T. Ortega (Eds.), Investigación en Educación Matemática XVIII (pp. 573-582). Salamanca: SEIEM

Cómo citar este artículo: Dávila Araiza, M. T. y Herrera Garcia, K. J. (2021). Reflexiones en torno al diseño de una propuesta formativa sobre variación lineal orientada a futuros profesores de secundaria. SAHUARUS. REVISTA ELECTRÓNICA DE MATEMÁTICAS. ISSN: 2448-5365, 5 (1), pp. 94-111. 